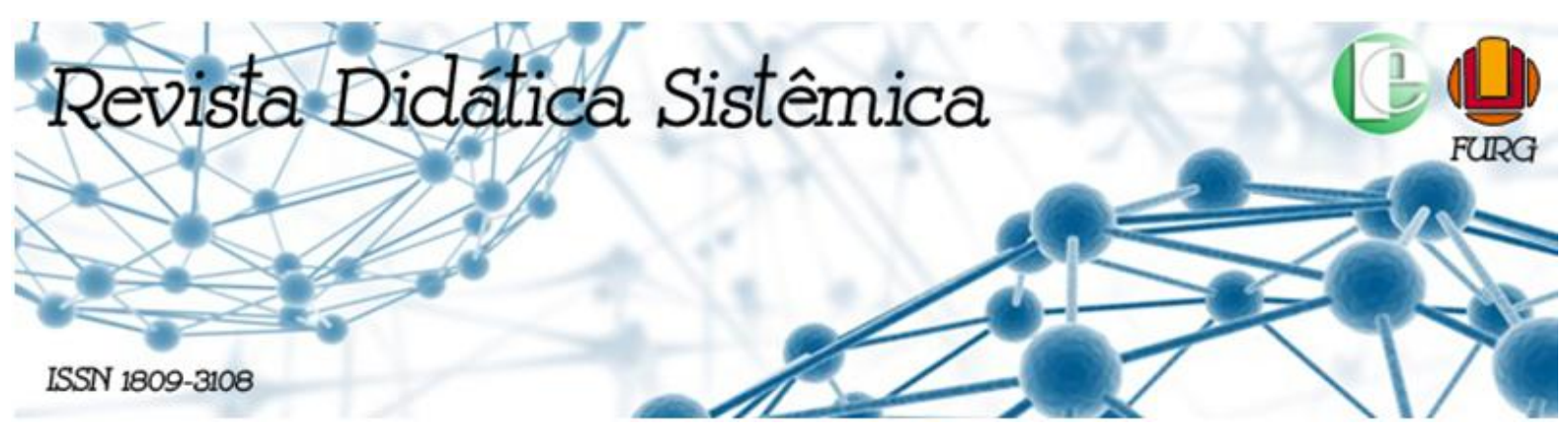

\title{
DE TELHADO EM TELHADO: O PARKOUR NA EDUCAÇÃO FÍSICA
}

\author{
César Augusto Otero Vaghetti* \\ Marcos Corrêa da Silva Borges*** \\ Eduardo Merino* \\ Dimitri Wuo Pereira ${ }^{* * *}$
}

\begin{abstract}
RESUMO
O objetivo foi apresentar os relatos de praticantes de Parkour em um projeto de extensão desenvolvido na Escola Superior de Educação Física/UFPel e discutir sua possibilidade de inserção no ensino de Educação Física. Esta pesquisa possui características de um estudo intervencionista qualitativo, resultante de projeto de extensão universitária. Quatro acadêmicos de Educação Física e nove membros da comunidade participaram do estudo. As intervenções ocorreram uma vez por semana, desenvolvidas por estudante do curso de bacharelado em Educação Física. Por meio de um questionário semiestruturado com dez perguntas abertas, os participantes afirmaram ser interessante a inserção do Parkour no ensino superior de Educação Física como uma nova oportunidade de trabalho, tanto como um novo método para aptidão física para o bacharel ou para o licenciado, quanto para o ensino superior enquanto disciplina acadêmica e cultura corporal.
\end{abstract}

Palavras-chave: Atividades de aventura. Free-running. Bacharelado. Licenciatura.

\section{DE TECHO EN TECHO: EL PARKOUR EN LA EDUCACÍON FÍSICA}

\section{RESUMEN}

El objetivo era presentar los relatos de los praticantes de Parkour en un proyecto de extensión desarrollado en la Escuela de Educación Física / UFPel, y discutir su posibilidad de inserción en la enseñanza de Educación Física. Esta investigacion tiene características de un estudio intervencionista cualitativo, resultado de un proyecto de extensión universitaria. Cuatro estudiantes de educación física y nueve miembros de la comunidad participaron en el estudio. Las intervenciones tuvieron lugar una vez a la semana, desarrolladas por un estudiante de la Licenciatura en Educación Física. A través de un cuestionario semi-estructurado con diez preguntas abiertas, los participantes declararon que era interesante incluir el Parkour en la educación superior en Educación Física como una nueva oportunidad de trabajo, tanto como un nuevo método de aptitud física para el profecional de educacion fisica, como para La educación superior como disciplina académica y cultura corporal.

Palavras-chave: Actividades de aventura. Free-running. Bacharelado. Licenciatura.

\footnotetext{
* Doutor em Educação em Ciências - Química da vida e saúde - FURG. Mestre em Ciências do Movimento Humano-Biomecânica - UDESC. Licenciado em Educação Física - UDESC. Professor adjunto Universidade Federal de Pelotas UFPEL

** Graduado Bacharel em Educação Física pela Universidade Federal de Pelotas. Instrutor de Parkour por 7 anos. Instrutor de defesa pessoal para PM e GM. Instrutor de musculação.

**** Doutor em Ciências da Saúde. Professor Adjunto na Universidade Federal de Pelotas.

***** Doutor em Educação Universidade Nove de Julho
} 


\title{
FROM ROOFTOP TO ROOFTOP: THE PARKOUR IN PHYSICAL EDUCATION
}

\begin{abstract}
The objective was to present the reports of Parkour's practitioners of an extension project developed at the School of Physical Education / UFPel, and to discuss their possibility of insertion in the teaching of Physical Education. This research has characteristics of a qualitative interventionist study, resulting from a university extension project. Four Physical Education students and nine community members participated in the study. The interventions took place once a week, developed by a Bachelor's student of Physical Education. A semi-structured questionnaire, with ten open questions, was used. The participants stated that it was interesting to include Parkour in higher education as a new job opportunity, both as a new method for physical fitness, and for higher education as an academic discipline and body culture.
\end{abstract}

Keywords: adventure activities. Free-running. Bachelor degree. Graduation.

\section{INTRODUÇÃO}

Derivado do Méthode Naturelle ${ }^{1}$ e do Parcours du Combattant ${ }^{2}$, o Parkour (PK) foi criado por David Belle, filho de Raymond Belle, o qual procurou preservar seus valores morais, como o incentivo à coragem e ao altruísmo através de exercícios corporais. Segundo Belle (2009), a ideia do PK é transpor obstáculos, tanto no meio natural quanto no urbano, tendo o corpo como único fundamento para resolver problemas do espaço em que se vive, sendo necessário preparar-se continuamente para tais feitos.

O PK se difundiu pelo planeta por variados meios de comunicação, como filmes cinematográficos $^{3}$, jogos digitais $^{4}$, propagandas de televisão ${ }^{5}$ e novelas ${ }^{6}$ (CLEGG \& BUTRYN, 2012). Como ponto de partida desta divulgação global, cita-se o filme de origem francesa, Banlieue 13, lançado em novembro de 2004 e estrelado por David Belle e Cyril Raffaelli, em que o PK é o pano de fundo de praticamente todas as cenas de ação e serviu para o lançamento mundial de Belle e de sua modalidade em grande escala.

Em Lisses, no subúrbio francês, Belle, seus primos e mais alguns amigos reuniam-se para treinar os movimentos aprendidos com seu pai, na década de 1980, de forma a divertir-se

\footnotetext{
${ }^{1}$ O Méthode Naturelle surgiu a partir de 1905 como uma proposta metódica, progressiva e contínua, para crianças e adultos proporcionando desenvolvimento integral (corpo e mente), tornando o ser humano um participante da sociedade forte e útil (SOARES, 2015).

${ }^{2}$ O Parcours du combattant é um treinamento militar originado do Méthode Naturelle, cujo objetivo é percorrer um percurso de obstáculos o mais rápida e eficientemente possível, com o objetivo de formar seres hábeis fisicamente. Foi criado pelo militar francês Raymond Belle.

${ }^{3}$ Exemplos de filmes: 007 Cassino Royale, Tracer, Assassin's Creed.

${ }^{4}$ Exemplos de jogos: Mirror's Edge, Dying Light, Vector.

${ }^{5}$ Exemplos de propaganda: Le petit Parkour (Antarctica), O percurso (Volkswagen), What would you do for a coke? (Coca-cola).

${ }^{6}$ Exemplo de novela: Malhação.
} 
e preparar-se para situações como as vividas por seu pai, um ex-combatente do Vietnã (CLEGG \& BUTRYN, 2012; PEREIRA et al., 2020).

Posteriormente, surge o Freerunning (FR), estilo de PK idealizado por Sebastién Foucan, um dos amigos de Belle, que se diferencia por demonstrar interesse maior à diversão e à plasticidade dos movimentos, com a adição de acrobacias como saltos mortais e outros movimentos tidos como desnecessários no PK (DERAKHSHAN \& MACHEJEFSKI, 2015).

Outra diferenciação do FR é seu cunho mais comercial (CLEGG \& BUTRYN, 2012), sua prática é voltada para demonstrações de habilidade, promoção da atividade, competitividade e lucro, o que os praticantes de PK não aceitam em sua filosofia original. Um bom exemplo disto são eventos internacionais de FR como o Red Bull Art of Motion, que ocorre anualmente desde 2007 em países distintos, e o Barclaycard Freerun World Championship, que estreiou em 2008, organizado pela Urban Freeflow ${ }^{7}$, com suas primeiras duas edições em Londres e a última, em Los Angeles, no ano de 2010.

Em seu livro, "Find your way", Foucan (2008) descreve o FR como a arte do movimento e da ação, permitindo que seus praticantes se expressem livremente em seu próprio ambiente, podendo ser adaptado de acordo com as características e habilidades de cada pessoa.

Atualmente, em nível mundial, o PK é representado pela Fédération Internacionale des Arts Du Déplacement (FIADD) e, nacionalmente, pela Associação Brasileira de Parkour (ABPK), criada em 2005.

Esta disciplina requer uma grande preparação física para os vários movimentos (saltos, vaults ${ }^{8}$, escalada, etc.) envolvidos, e também é necessária uma mente decidida a superar seus próprios medos, uma concentração elevada, e um espírito forte (SOTO et al., 2013, p.58).

O PK é uma atividade de aventura, também conhecida como a arte do deslocamento, ele objetiva a transposição de obstáculos de forma rápida e eficiente. Segundo Alves e Corsino (2013) o PK é um esporte radical de aventura, porém na literatura

\footnotetext{
${ }^{7}$ A Urban Freeflow, fundada por Paul Corkery em 2003, foi a primeira marca relacionada ao $P K$ e ao $F R$, seu objetivo era formar uma comunidade internacional de praticantes com fóruns para debate e discussão. Com o sucesso do empreendimento, houve diversas participações em filmes e documentários como 007 Cassino Royale, Devil's Playground e Punisher War Zone, Jump Britain, The way e Planet Parkour. A Urban Freeflow divide opiniões entre os praticantes por se autointitular a Rede Mundial Oficial do $P K$ e $F R$ e polemiza por apoiar versões competitivas em campeonatos mundiais patrocinados pela Barclaycard (BAVINTON, 2007).

${ }^{8}$ Vaults são técnicas utilizadas no Parkour para transpor rapidamente obstáculos, com saltos e passagens em apoio. Existem vários tipos de vaults, cada um para diferentes tipos de obstáculo, velocidade e direção do movimento.
} 
especializada pode-se encontrar três formas diferentes, como esporte, como disciplina e como arte.

Segundo os relatos de um praticante no estudo de Bavinton:

Pode ser considerado os três, depende do indivíduo, para mim, é mais como um esporte, se eu estivesse mais profundamente envolvido no Parkour poderia vir a ser uma disciplina para mim e se eu fosse tão bom quanto David Belle talvez fosse uma arte também (2007, p. 401).

Um outro apontamento semelhante é descrito por Marques (2010), o qual também caracteriza o PK como estilo de vida, como arte urbana e como filosofia de treinamento. Abellán-Aynes e Alacid (2016) também afirmam que a prática do PK é uma atividade recreacional extrema onde seus praticantes superam obstáculos com rapidez e fluidez, correndo, saltando, transpondo obstáculos e escalando. Uma descrição diferente é defendida por Chagas et al., (2016) o qual defendem que os princípios que fundamentam o PK como uma disciplina são as aplicações dessas habilidades em uma formação para a vida, onde o sujeito torna-se útil para adaptar-se a qualquer ambiente e circunstância.

Uma das definições mais interessantes sobre o PK é relatada por Clegg e Butryn (2012) os quais referem-se ao PK como a arte da aventura urbana, de origem francesa em que seus praticantes têm por objetivo percorrer um percurso adaptando suas técnicas para transpor os obstáculos de forma eficiente, rápida e segura, utilizando apenas o corpo. O PK é um método em que o praticante deve ter como objetivo tornar-se autônomo fisicamente, sendo capaz de salvar a si mesmo e a outros em situações de perigo, apenas com a utilização das habilidades corporais adquiridas com a prática (STRAMANDINOLLI et al., 2012). Além disso, no PK há uma forte preocupação com a eficiência no movimento (DERAKHSHAN \& MACHEJEFSKI, 2015) no sentido de poupar energias para os obstáculos que ainda estejam por vir. Segundo Silva et al. (2010), as práticas corporais na natureza podem ser classificadas de acordo com o risco que apresentam, esta classificação divide-se em três categorias denominadas risco-perigo, risco-probabilidade e risco-aventura. Estes são os preceitos que localizam o PK dentro das atividades de aventura: a procura pelo inusitado, pela emoção e a forma como os praticantes lidam com os riscos que os colocam em situações em que não há retorno possível.

Diante das exigências físicas da modalidade, o PK apresenta-se como uma promissora ferramenta pedagógica para a EF, como conteúdo curricular nas escolas, para o ensino da modalidade como atividade recreacional e de aventura, e na sua utilização 
como método para desenvolver aptidão física. Porém, como apontam Leite et al. (2011), o PK é uma prática relativamente nova no país e pouco conhecida e utilizada pelos profissionais de Educação Física.

Tendo em vista as possibilidades para a inserção do PK em diferentes âmbitos relacionados à EF, esta pesquisa tem como objetivo apresentar os relatos de praticantes de PK em um projeto de extensão desenvolvido na Escola Superior de Educação Física da UFPel, e discutir a possibilidade de inserção do PK no ensino de Educação Física para os cursos de bacharelado e licenciatura.

\section{PROCEDIMENTOS METODOLÓGICOS}

Esta pesquisa possui características de um estudo intervencionista qualitativo, resultante do projeto de extensão universitária "Parkour na Educação Física: Uma introdução à modalidade", pensado a partir das ações de ensino realizadas nas disciplinas Atividades Físicas de Ação na Natureza, Esportes de Aventura, e Educação Física e Meio Ambiente.

A divulgação do projeto deu-se através de cartazes dispostos na ESEF/UFPel e no campus Anglo, e também através da rede social Facebook. As inscrições foram realizadas na secretaria da ESEF/UFPel. As aulas/intervenções tiveram duração de 24 semanas, contando com uma intervenção por semana com carga horária de 3 horas. Além do conteúdo prático, foi realizada uma palestra sobre os referenciais históricos e filosóficos da modalidade para os participantes no primeiro dia do projeto. Os participantes foram estimulados ao diálogo, expondo suas dúvidas e questionamentos neste momento.

O projeto foi realizado no ginásio da ESEF/UFPel, e no Parque da Baronesa, ambos na cidade de Pelotas, durante o segundo semestre de 2016. Nas intervenções no ginásio, utilizou-se materiais da GA: plintos, traves, steps, barras assimétricas, colchonetes e colchões. No Parque da Baronesa, foi utilizada a própria estrutura arquitetônica do local.

Os públicos alvo foram acadêmicos de Educação Física que tivessem interesse na modalidade, e comunidade em geral. Como requisito para a participação, pedia-se apenas que os interessados fossem maiores de 15 anos de idade. Participaram do estudo 13 indivíduos dos quais, 4 acadêmicos da ESEF/UFPel e 9 da comunidade. A idade dos participantes variou entre 15 e 28 anos.

As aulas práticas foram organizadas de forma a apresentar uma progressão metodológica das técnicas do PK, começando com a corrida e o sprint (corrida de alta 
velocidade e curta duração), os saltos de precisão ${ }^{9}$, a quadrupedia (técnica básica de se deslocar em quatro apoios) e o cat balance ${ }^{10}$.

Posteriormente, foi possibilitada a familiarização dos participantes com os percursos e aparelhos utilizados, deixando que transitassem entre eles e apontassem formas de transpor seus obstáculos e, em seguida, apresentados os primeiros vaults básicos. Inicialmente, os vaults foram trabalhados separadamente e utilizando-se de variações dos movimentos, quando possível, e de forma fracionada, até chegar ao movimento final.

$\mathrm{Na}$ etapa seguinte do projeto, foi proposto aos participantes que percorressem diferentes percursos, utilizando vaults pré-selecionados para cada obstáculo. Na penúltima intervenção foi montado um percurso e proposto que os participantes o percorressem diversas vezes, tendo total liberdade para utilizar quaisquer técnicas apresentadas anteriormente, objetivando despertar a sua autonomia e a autoconfiança, bem como observar os resultados da relação ensino-aprendizagem alcançados com o projeto.

$\mathrm{Na}$ última intervenção os participantes foram convidados a praticar nas dependências do Parque da Baronesa. Esta intervenção se destacou das demais pelo entusiasmo dos participantes, pela descontração nas práticas. Também, pode-se observar o que conseguiram aplicar do que aprenderam em um ambiente menos controlado, em contato com a areia e paredes de tijolos, em que o PK realmente ganha forma e expressividade.

Ao término do projeto, aplicou-se um questionário semiestruturado, composto por 10 perguntas abertas, desenvolvido especificamente para avaliação do projeto. Além de procurar responder à questão norteadora do estudo, o questionário visou também uma caracterização dos participantes, bem como identificar alguns aspectos relevantes que poderiam auxiliar na elaboração de novos projetos voltados ao PK.

\section{RESULTADOS E DISCUSSÃO}

Com relação ao projeto de extensão, deve-se levar em consideração que, por ser um estudo qualitativo de caráter sociocultural, não comporta generalizações. Suas bases

\footnotetext{
${ }^{9}$ Segundo Puddle e Maulder (2013), chama-se "salto de precisão" a técnica utilizada para amortecer o pouso apenas com a porção frontal dos pés, flexionando os joelhos para absorver e utilizando os braços para contrabalancear o movimento.

${ }^{10}$ Cat balance é uma técnica utilizada para deslocar-se em cima de muros em quatro apoios. Diferente da quadrupedia, onde braços e pernas deslocam-se ao lado do corpo, no cat balance, pernas e braços deslocam-se na linha medial do corpo
} 
analógicas baseiam-se na descrição, análise e interpretação das informações recolhidas durante o processo de intervenção com a prática do $\mathrm{PK}$, sendo possível, apenas, um entendimento pleno de forma contextualizada.

A implementação do PK no cotidiano dos acadêmicos da ESEF/UFPel teve como intuito oferecer uma modalidade distinta das ofertadas pela instituição, de forma a ampliar o leque de opções de vivências corporais, e, ainda, introduzir uma filosofia diferente, baseada no incentivo à coragem, ao altruísmo e num pleno desenvolvimento físico, características não obstantes da fundamentação da própria Educação Física.

Assim como o surf e o skate, o PK surge como uma nova opção para os acadêmicos, do ponto de vista profissional e didático, diferenciando-se das demais disciplinas que envolvem esportes convencionais, podendo ser implementado à disciplina de Atividades Físicas de Ação na Natureza ou de Esportes de Aventura.

De acordo com as respostas obtidas com o questionário, os quatro participantes acadêmicos afirmam ser interessante inserir o PK no ensino superior de Educação Física. Segundo Stramandinoli et al. (2012), é natural que o PK possa ser inserido como objeto de estudos da Educação Física, pois o PK é a arte de se movimentar no espaço e a Educação Física tem o compromisso de estudar o homem em movimento. Considera-se que a Educação Física deve colaborar na organização didática e metodológica do PK, pois este é um dos fundamentos da profissão.

Essa atividade se preocupa com a prevenção das lesões, mas não traz de forma organizada uma metodologia de ensino sistematizada e com isso o processo de aprendizagem em grupos de praticantes é diferente de academias, clubes, ou outros espaços de convivência social em que o esporte costuma ser protagonista, levando também professores de Educação Física a ensinar a modalidade, talvez de forma equivocada em relação à filosofia inicial (PEREIRA; HONORATO; AURICCHIO, 2020, p. 149).

Sobre a aplicabilidade da prática do PK no ambiente acadêmico nas áreas de bacharelado, três participantes entrevistados apontaram ser uma nova oportunidade de trabalho; e um, como ferramenta auxiliar de outras práticas. F.R. comenta que: "O bacharel tem que aproveitar a oportunidade de uma área nova para se investir, abrindo estúdios ou academias focadas na prática do Parkour".

B.C.D. diz: "É uma metodologia de trabalho que pode ser utilizada para a obtenção de uma melhora do condicionamento físico. Os conhecimentos adquiridos durante a graduação facilitam o ensino do Parkour". 
$\mathrm{Na}$ área da licenciatura, dois participantes apontaram o PK como uma ferramenta pedagógica interessante para desenvolver as capacidades motoras das crianças e como uma atividade diferenciada de prática que pode ser proposta no ambiente escolar.

C.S.A. salienta que: "Com as dificuldades encontradas na realidade das aulas nas escolas, ter uma atividade nova, estimulante, e que praticamente não necessita de materiais para a sua prática, é interessante".

P.D.M. acredita que: "O Parkour é interessante para aumentar e melhorar o repertório motor das crianças". Esta afirmativa pode ser encontrada na referência de Carvalho e Viana (2017) os quais afirmam que o PK pode enriquecer as aulas e aprimorar as funções motoras dos alunos, sendo uma experiência motora variada.

Dos participantes, $80 \%$ acreditam ser necessário um bom condicionamento físico para a prática do PK, em concordância com os apontamentos de Soto et al. (2013, p. 58), "Esta disciplina requer uma grande preparação física para os vários movimentos (saltos, vaults, escalada, etc.) envolvidos [...]".

Quando questionados sobre a prática do PK auxiliar na melhora do condicionamento físico, a resposta positiva foi unânime, corroborando com os resultados encontrados por autores como Nagata e Do Carmo (2012), que apontam os benefícios do PK para o ganho de potência de membros inferiores e desenvolvimento motor; Carvalho e Viana (2017), apontando para os benefícios relacionados à aptidão física e desenvolvimento motor; e Leite et al. (2011), relacionando à prática do PK com o desenvolvimento de membros superiores, melhora da força explosiva de membros inferiores, tanto para salto vertical quanto salto horizontal.

E.O.D. diz: “Com certeza. É uma atividade que exige um bom preparo físico mesmo para as atividades mais básicas".

No estudo de Tremblay e Willms (2003), aponta-se que crianças canadenses tendem a praticar esportes não convencionais por mais tempo que os convencionais, produzindo um impacto mais profundo na diminuição da obesidade infantil. Já na França, o aumento da participação nos esportes está diretamente relacionado com este tipo de atividade, e, entre $45 \%$ e $60 \%$ da população, praticam algum esporte não convencional (L'AOUSTET \& GRIFFET, 2001). A prática do PK é uma forma desafiadora de movimentar-se e este aspecto é bastante apreciado pelas crianças e jovens (PEREIRA \& ARMBRUST, 2010).

C.B.S. comenta: "Sim. A prática melhora o fôlego, a resistência e também o tempo de reação". Sobre este último, pode-se apontar a seguinte referência: 
O PK pode ser um tema de fundamental importância para a Educação Física Escolar, pois, além de colocar em prática as habilidades em situações reais, pode simular situações de perigo, desenvolve habilidades tanto na esfera da motricidade quanto na cognitiva, pois dessa forma, ao assumir riscos, mesmo que de modo controlado, o aluno deverá resolver situações problemas que dentre outras coisas, exercitará seu tempo de reação, senso de iniciativa e rapidez de adaptação, características essas que estimulam uma postura crítica e de tomada de decisões (ALVES \& CORSINO, 2013 p. 254).

Seis dos dez entrevistados já haviam praticado PK anteriormente ao projeto.

Citando C.A.S.: "Eu já praticava e tinha acabado de entrar para a ESEF. Queria continuar meus treinos e estava interessado na inserção do Parkour na Educação Física".

Os entrevistados interessaram-se pelo projeto e inscreveram-se nele, principalmente, (60\%) para aprender ou aperfeiçoar as técnicas básicas da modalidade. O mesmo número relatou que a prática em ambiente controlado (dentro do ginásio) foi importante pela questão da segurança, mas que essa vivência, por si só, não foi suficiente para abranger as possibilidades do PK como modalidade essencialmente urbana.

Como cita J.A.: “Eu gostei da prática no ginásio por iniciar em um ambiente seguro, mas senti que falta a vivência de uma prática no ambiente urbano, para praticar o Parkour como realmente é".

Outro comentário interessante é de C.B.S.: “A prática em um ambiente seguro deve ser considerada treino. A situação real é quando se pratica na rua. Ambos são necessários".

É importante ressaltar que o quesito "segurança" é citado por $60 \%$ dos entrevistados como um dos fatores positivos de praticar nas dependências da ESEF/UFPel. Em resposta à questão referente à motivação para inscrever-se no projeto, B.C.D. responde: “Um ambiente seguro e didático para a aprendizagem".

Diversão foi outro ponto apontado por $30 \%$ dos participantes entrevistados, corroborando com resultados encontrados no estudo de Clegg e Butryn (2012), no qual os sujeitos apontam a diversão como sendo um dos elementos fundamentais e universais na prática do PK:

Um elemento fundamental do jogo é se divertir. Para esses participantes, a sensação de se divertir ao praticar, treinar ou realizar o parkour foi universal. [...] Eu não estou fazendo isso apenas para vencer o percurso, eu estou fazendo para me divertir com isso. E no segundo que você para de se divertir com isso, então, não faz mais sentido (CLEGG \& BUTRYN, 2012 p. 327).

De acordo com a literatura apontada neste trabalho, o PK apresenta grande potencial para ser trabalhado em âmbito da Educação Física, principalmente no meio escolar, 
em todo o ensino básico. Nas séries iniciais, no sentido de que traz inúmeros benefícios motores e psicossociais para estes alunos, e, nas séries finais, contribui para a diversidade de conteúdo, estimulando o interesse dos jovens nas aulas de Educação Física com atividades diferenciadas e desafiadoras (ALFREDO \& PEREIRA, 2011).

As limitações encontradas durante a elaboração do estudo foram: a falta de uma maior divulgação do projeto, resultando num baixo número de participantes, tornando inviável uma resposta mais precisa com relação ao escopo do estudo; a falta de tempo dos acadêmicos interessados devido às responsabilidades da faculdade e a impossibilidade de realizar as práticas em local apropriado para uma vivência mais abrangente e próxima da prática real de PK.

Futuros estudos com um maior número de participantes e com disponibilidade de locais externos, com possibilidades distintas para a prática, são necessários para uma abordagem mais significativa da modalidade e para a obtenção de informações mais precisas. Um outro ponto relevante é, a partir das experiências deste estudo, que um novo questionário específico mais aprofundado e preciso seja elaborado para novos estudos sobre o tema.

\section{CONSIDERAÇÕES FINAIS}

De acordo com a análise das respostas obtidas no questionário, o PK teve uma boa aceitação no ambiente acadêmico do curso de Educação Física da ESEF/UFPEL. O PK foi relatado pelos praticantes como uma prática promissora, que pode acrescentar conhecimentos para formação acadêmica dos alunos. A oferta dessa prática no ambiente acadêmico também demonstrou resultados positivos no sentido de disponibilizar um espaço em que a comunidade possa praticar, de forma segura e orientada, por meio de projetos de extensão.

$\mathrm{Na}$ área do bacharelado, o PK pode ser utilizado como um método para o desenvolvimento da aptidão e do condicionamento físico. Com relação à licenciatura, o PK possibilita um trabalho físico sem a necessidade de implementos esportivos, facilitando sua implementação nas escolas. Além disso, o PK também deve ser estimulado como uma prática com fim em si mesmo, ou seja, a própria prática da aventura urbana.

\section{REFERÊNCIAS}

ABELLÁN-AYNÉS, O; ALACID, F. Anthropometric profile, physical fitness and differences between performance level of Parkour practitioners. Archivos de Medicina del Deporte, p. 312-316, 2016. 
ALFREDO, J., PEREIRA, D. W. Le Parkour. In: BERNARDES, L. A. Atividades e esportes de aventura para profissionais de Educação Física. São Paulo: Phorte, 2011.

ALVES, C. S. R.; CORSINO, L. N. O Parkour como possibilidade para a Educação Física Escolar. Motrivivência, n. 41, p. 247-257, 2013.

BAVINTON, N. From obstacle to opportunity: Parkour, leisure, and the reinterpretation of constraints. Annals of Leisure Research, v. 10, n. 3-4, p. 391-412, 2007.

BELLE, D. Eu salto de telhado em telhado. Entrevista, 2009. Disponível em https://www.youtube.com/watch?v=myuX_qQATa8. Acessado em: 12 mai. 2017.

CARVALHO, W. B. G. R.; VIANA, J. B. R. A avaliação do desenvolvimento da aptidão física através da prática do Parkour no âmbito da educação física escolar. ACTA Brasileira do Movimento Humano, v. 6, n. 2, p. 20-33, 2017.

CHAGAS, R. R; ROJO, J. R; GIRARDI, V. L. O processo de esportivização de uma modalidade: O Parkour enquanto prática espetacularizada. The Journal of the Latin American Socio-Cultural Studies of Sport (ALESDE), v. 5, n. 1, p. 21-33, 2016.

CLEGG, J. L.; BUTRYN, T. M. An existential phenomenological examination of Parkour and Freerunning. Qualitative Research in Sport, Exercise and Health, v. 4, n. 3, p. 320-340, 2012.

DERAKHSHAN, N; MACHEJEFSKI, T. Distinction between Parkour and Freerunning. Chinese Journal of Traumatology, v. 18, n. 2, p. 124, 2015.

FOUCAN, S. Free Running: Find Your Way. London: Michael O'Mara, 2008.

L'AOUSTET, O.; GRIFFET, J. The Experience of Teenagers at Marseilles' Skate Park: Emergence and Evaluation of an Urban Sports Site. Cities, v. 18, n. 6, p. 413-418, 2001.

LEITE, N. et al. Perfil da aptidão física dos praticantes de Le Parkour. Revista Brasileira de Medicina do Esporte, v. 17, n. 3, p. 198-201, 2011.

MARQUES, R. A. Cidade lúdica: um estudo antropológico sobre as práticas de Parkour em São Paulo. Tese de Doutorado. Universidade de São Paulo, 2010.

NAGATA, E. Y.; DO CARMO, T. A. A adoção do Le-Parkour como meio de desenvolvimento de força em membros inferiores em alunos do ensino fundamental. IX encontro de iniciação científica e VII mostra de pós-graduação. Anais..., 2012.

PEREIRA, D. W.; ARMBRUST, I, Pedagogia da Aventura: os esportes radicais, de aventura e ação na escola. Jundiaí - SP: Fontoura, 2010.

PEREIRA, D. W.; HONORATO, T.; AURICCHIO, J. R. Parkour do princípio filosófico ao fim competitivo. Belo Horizonte: Licere, v. 23, n. 1, p. 134-152, mar. 2020.

PUDDLE, D. L.; MAULDER, P. S. Ground reaction forces and loading rates associated with Parkour and traditional drop landing techniques. Journal of Sports Science and Medicine, v. 12, n. 1, p. 122-129, 2013.

SILVA, P. P. C. et al. Risco e práticas corporais na natureza: uma revisão sistemática. Revista Brasileira de Ciência e Movimento, v. 18, n. 2, p. 84-91, 2010.

SOARES, C. L. Uma educação pela natureza: O método de Educação Física de Georges Hébert. Revista Brasileira de Ciências do Esporte. v. 37, n. 2, p. 151-157, 2015.

SOTO, J. J. P. et al. Desarrollo de las capacidades coordinativas a través del juego: Parkour. 2013.

STRAMANDINOLI, A. L. M.; REMONTE, J. G.; MARCHETTI, P. H. Parkour: História e Conceitos da Modalidade. Revista Mackenzie de Educação Física e Esporte, v. 11, n. 2, p. 13-25, 2012.

TREMBLAY, M S; WILLMS, J D. Is the Canadian childhood obesity epidemic related to physical inactivity? International Journal of Obesity, v. 27, n. 9, p. 1100, 2003. 